\title{
STUDY OF PARTICLE IN CYCLONES
}

\author{
M. Valverde - Ramirez and J. A. S Gonçalves \\ Federal University of São Carlos \\ Rodovia Washington Luís, km 235 - SP 310 \\ São Carlos - Brazil \\ valverderamirez@hotmail.com and jasgon@ufscar.br
}

\begin{abstract}
The turbulent gas - particle flows in cyclone was simulated considering the transfer of momentum by one-way coupling and two-way coupling. the cyclone diameter was $0.3 \mathrm{~m}$. mono-dispersed particle in diluted flow was injected. the size of the particle injected in the cyclone were $1-5 \mu \mathrm{m}$. the inlet gas velocity was $20 \mathrm{~m} / \mathrm{s}$. the euler - lagrange modeling approach has been was used in the simulate. the turbulence model used was the reynolds stress model, which has been reported to give good results for cyclones. particle trajectories were calculated by the discrete phase model. simulations were made for both one-way (when the gas movement is not affected by the presence of the articles) and two-way coupling (when both phases influence each other). the efficiency of collection, tangential velocity and axial velocity were obtained for both coupling schemes. the results show the some behavior for the mean tangential velocity and mean axial velocity when are simulated using with one-way coupling and two-way coupling. the collection efficiency has a light variation between one-way and two way coupling. the numerical data were agreement with the experimental data.
\end{abstract}

Keywords: cyclone, one-way coupling, two-way coupling, efficiency of collection.

\section{INTRODUCTION}

Turbulent flow can be used to separated particles with different density, different size. The phenomena that govern the operation of the cyclones are complex; this is why the understanding of the process of particles separation is still not properly understood. In the cyclone, are present phenomena such as turbulence, vortex, reverse flow, regions with ascending and descending flow which produce instability and asymmetry in the flow. The quantification of the cyclone performance is carried out through the calculation of collecting efficiency and pressure drop (Hoffman and Stein, 2002).

According Derksen (2003), Derksen et al. (2006), Derksen et al. (2008) and Utakir et al. (2010) turbulence in the cyclone plays an important role in the process of separation of the particles. The particle can modify the formation of swirls and the turbulence intensity of the continuous phase. The swirls and turbulence are reduced with the increasing mass of the particle (Derksen et al., 2008). Derksen et al. (2006) and Derken et al. (2008) observed that the presence of particles reduced the intensity of the turbulence and swirls and creates a strong damping of the turbulence; swirls intensity decreased more in the lower part of the cyclone. The pressure drop decreases steadily with increasing of the mass while the collection efficiency has complex response with the mass increasing. Wan et al. (2008) observed that with the increase of mass concentration, the turbulent intensity decreased.

The introduction of the concept of the relationship between the volume fraction and the type of coupling was introduced by Elghobashi et al. (1991). Dilute gas - particle flows are characterized 
by a volume fraction $\Phi_{v}$ smaller than $10^{-5}$, the continuous phase has a predominant effect on the dynamics of turbulence and transport of the dispersed phase is considered one coupling. When gas particle has fraction volumetric between $10^{-5} \leq \Phi_{v}<10^{-3}$, the particle changes the dynamics of turbulence and two-way coupling should be considered in the simulation.

One-way coupling offers an insight in basic physics of particle-laden turbulent flow (L'vov et al., 2003).Two-way coupling considers momentum transfer from dispersed phase to continuous phase through appropriate source terms in the momentum, Reynolds-stress or turbulent kinetic energy and dissipation rate equations (Crowe et al., 2012).

According to Loth (2003), in diluted flows the particle motion is controlled by the continuous phase but when the particle hits the wall or other particle, there is the effect of the impact on the system even at volume fractions of particles below $0.1 \%$. The author indicates that there is no consensus on how to incorporate the effect of dispersed phase on the continuous phase diluted.

Shukla et al. (2011) observed that many study numerical in cyclones discussed about mean velocity profiles while the prediction of velocity fluctuation is least studied, possibly due to application of steady solver in prediction of flow field characterization of cyclone. The mean velocity distribution is a key parameter because it strongly influenced the pressure distribution (Gimbun, 2008) and show that the centrifugal field, depend by the internal geometry layout and sensitive condition downstream the cyclone (Hoekstra et al., 1999). Effect of the velocity fluctuation it is important parameter in separation of particle from 0.6 to $10 \mu \mathrm{m}$ (Gimbun, 2008 and Shukla et al., 2013).

Mallouppas and Wachem (2013) concluded that for dilute turbulent gas - particle flow, there are difference between the results obtained by the one-way coupled and two-way coupled models, having a particularly large effect over the particle velocity fluctuation. L'vov et al. (2003) indicate that particle can modified the turbulence increasing or reducing gas fluctuation velocity in shear flows. Prediction of particle motion requires an accurate estimation of turbulent behavior and velocity fluctuation (Utakir et al., 2010).

The turbulent motion of the gas - particle flow is composed by the mean flow and the fluctuation flow. The fluctuation influence the mean flow through the Reynolds stresses while the mean flow also influences the fluctuation since the turbulent production is proportional to the mean velocity gradient and the Reynolds stresses (Tennekes and Lumley, 1972).

In this study, is investigated the difference and effects of one-way coupled and two-way coupled on simulations. It were injected mono-dispersed particle with size $1-5 \mu \mathrm{m}$. The simulations in this article were performed using the package FLUENT 14.5.

\section{OVERVIEW OF NUMERICAL SIMULATION}

The numerical approximation of the flow gas - particle is approached by Euler-Lagrange technique. The simulated particles in the cyclone are inert. The coupling to the gas phase with the particle phase was made using one-way coupling and two-way couplings and was used Reynolds Stress turbulent model. It is considered that there is no collision between the particles.

\subsection{Momentum and mass conservation equations}

Euler representation of fluid motion considering the mass and momentum conservation Equations. 


$$
\begin{aligned}
& \boldsymbol{\nabla} \cdot \boldsymbol{u}=0 \\
& \rho\left[\frac{\partial \boldsymbol{u}}{\partial t}+(\boldsymbol{u} \cdot \nabla) \boldsymbol{u}\right]=-\nabla p+\mu \nabla^{2} \boldsymbol{u}+\boldsymbol{f}^{p}
\end{aligned}
$$

where $\boldsymbol{u}$ is velocity vector, $p$ is the pressure, $\rho$ the density, $\mu$ the dynamic viscosity of the fluid. In two-way coupled simulation, the term $\boldsymbol{f}^{p}$ is added in the momentum equation and represents the feedback force per unit volume due to the presence of the particles. The gas motion, instantaneous velocity $u(\tau)$ is composed by the mean flow $(\bar{u})$ and the fluctuations $\left(u^{\prime}\right)$. The interaction between two phases are linked by the drag force. The body force $f^{p}$ is the reaction force from the particles onto the fluid.

$$
\boldsymbol{u}(\tau)=\overline{\boldsymbol{u}}+\boldsymbol{u}^{\prime}
$$

The turbulent term $\left(-\rho \overline{u_{\imath} u_{\jmath}}\right)$ which represent additional shear stress components caused by the fluctuating velocity is modeled. The transport equation of Reynolds stress tensor in the Reynolds Stress turbulent model is described by the following equation:

$$
\frac{D\left(\rho \overline{u_{\imath}^{\prime} u_{\jmath}^{\prime}}\right)}{D t}=\boldsymbol{P}_{i j}+\boldsymbol{B}_{T, i j}+\boldsymbol{D}_{L, i j}+\emptyset_{i j}-\varepsilon_{i j}
$$

where $\boldsymbol{P}$ is the production due to mean shear, $\boldsymbol{B}$ is the production due to particle-fluid interaction forces, $\boldsymbol{D}$ is the turbulent and viscous diffusion, $\varnothing$ is the pressure-strain interaction, and $\varepsilon$ is the viscous energy dissipation.

The Lagrange representation of the motion of particle.

$$
\begin{aligned}
& \frac{d x_{\boldsymbol{p}}}{d t}=\boldsymbol{v}_{\boldsymbol{p}} \\
& m_{p} \frac{d V}{d t}=\sum_{i} \boldsymbol{F}_{i}
\end{aligned}
$$

where $x_{p}$ is the location where the particle, $m_{p}$ is the mass of the particle motion, $F_{i}$ the total force over the particle. The drag force is considered to be the dominant force in the cyclone (Utakir et al., 2010 and Shukla et al., 2011a and b).

$$
\begin{aligned}
& \boldsymbol{F}_{D}=\boldsymbol{F}_{\text {surf }}+\boldsymbol{F}_{\text {body }}=2 \pi \mu a v_{p}+4 \pi \mu a v_{p}=6 \pi \mu a \boldsymbol{v}_{\boldsymbol{p}} \\
& C_{D}=\frac{F_{D}}{\mu v_{p} a}=6 \pi
\end{aligned}
$$

An alternative widely used to calculate the drag coefficient $C_{D}$ are correlations proposed by Morsi and Alexander in 1972. The particle trajectory calculation are carried out through numerical integration of Equation (5) and Equation (6).

$$
\boldsymbol{v}_{p}(t)=\overline{\boldsymbol{v}}_{p}+\boldsymbol{v}_{p}^{\prime}
$$

The discrete random walk model has been used to model the effect of fluid turbulence on the particle path. This model show a tendency for such particles to concentrate in low-turbulence 
regions of the flow. The values of the fluctuation of the velocity follow a Gaussian probability distribution (Fluent User Manual).

$$
\boldsymbol{v}_{p}^{\prime}=\zeta \sqrt{\overline{\boldsymbol{v}_{p}^{\prime 2}}}
$$

where $\zeta$ is a normal distributed random number. The Euler fluid equation and the Lagrange particle equation are integrated forward in time with the same time step. In two-way coupling each and every particle acts back onto the local fluid with a point force. Interpolation from the particle centers to the mesh. The particle source terms $\boldsymbol{f}^{p}$ have a different impact on the flow and on the particle. The approach two way coupling has two different time steps, corresponding to the particle and the fluid (Fluent User Manual).

$$
\boldsymbol{f}^{p}=-\frac{1}{V_{c e l l}} \sum_{l=1}^{n p} \boldsymbol{F}_{l}
$$

where the subscript $l$ refers to the particle number, $n p$ the total number of the particles in a fluid, $V_{\text {cell }}$ is cell volume. The source term $\boldsymbol{f}^{p}$ is calculated using the concept "Particles - Source- InCell" method. Derksen et al. (2006); Derksen et al. (2008) and Wan et al. (2008) used the Lagrange approach with this method and to simulate the coupling of the phases in the cyclone.

\section{SIMULATION SET-UP}

The simulation are performed in the Euler - Lagrange and framework and turbulence Reynolds stress model. The numerical and the experimental data were compared. Other authors have also used these experimental data as a basis of comparison were Zhao et al. (2006); Shukla et al. (2011a); Shukla et al. (2011b), and Shukla et al. (2013).

The cyclone geometry and distribution of the cells are shown in Figure 1. The cyclone dimensions were taken from the experimental work of Zhao et al. (2004). The diameter of the cyclone was $0.3 \mathrm{~m}$. For the construction of the computational mesh geometry of the cyclone was divided into blocks. In each block was made the decomposition of the domain into cells.

The mesh was refined in the central region of the vortex core and the regions near the wall. In the simulations were used approximately 750000 cells. Previously been made stability analysis mesh. The results of pressure drop remained constant.

The boundary conditions used were velocity inlet $(20 \mathrm{~m} / \mathrm{s})$, pressure outlet $(0 \mathrm{pa})$, no slip condition into the continuous phase walls. To the dispersed phase is regarded as the coefficient of restitution equal to 1 in almost all internal surfaces of the cyclone, except the bottom surface of the collector where it was considered that if the particle reaches this area will be retained. In other walls, after contact with the wall, the particle returns to flow without energy loss.

The algorithm used for the pressure-velocity coupling was the type SIMPLE. The discretizations used were the type PRESTO for pressure and type QUICK for the moment. The convergence criterion used was the difference in residues of the equations of continuity <1x10-4. The total number of injected particles was 900 units. The diameter of injected particle were 1 to 5 $\mu \mathrm{m}$. The concentration of injected solid material was, $0001 \mathrm{~kg}$ of particle/s. The integration scheme of the particles was the type trapezoid. The time step used was equal to $0.001 \mathrm{~s}$. 


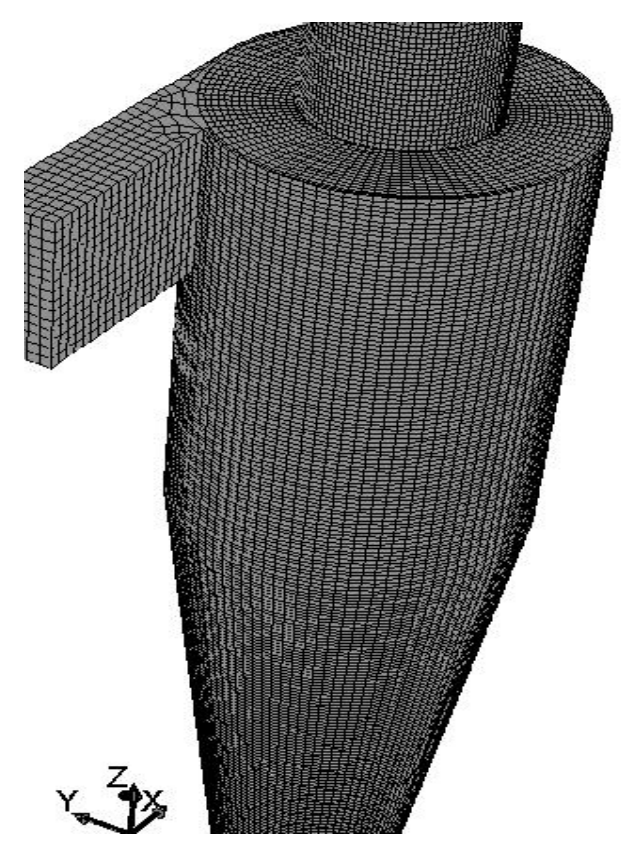

Figure 1 - Cyclone.

\section{RESULTS AND DISCUSSION}

The numerical results of the simulation are compared with the experimental results obtained by Zhao et al. (2004) and Zhao et al. (2006). Figures 2, 3 and 4 show numerical data (one-way coupling, two-way coupling) and experimental data. Dilute system and simulation transients were used in this study.

The reading of the mean tangential velocity and mean axial velocity was done on a horizontal line located in the middle of the cylindrical surface $(\mathrm{z}=0.67 \mathrm{D}$, and $\mathrm{D}=0.3 \mathrm{~m})$. The same height was considered by Zhao et al. (2006).

Figures 2 and 3 compares the velocity (tangential and axial) obtained by simulating whit experimental data. It is observed the same behavior in one-way coupling simulation and two-way coupling. This means that the average mean fluid velocity is not influenced by the presence of the particles.

Derksen et al. (2006) and Wan et al. (2008) shows changes in the velocity when simulate with one-way coupling and two-way coupling. The authors observed reduction in the intensity of the tangential velocity on two-way coupling compared with one way coupling. Also observed that the axial velocity reduction (near the wall) and increment (in the vortex core region) changes oscillatory.

Derksen et al. (2006) used turbulence model large-eddy simulation and injected $10^{7}$ particles in the cyclone. Wan et al. (2008) used turbulence Reynolds stress model and injected $10^{7}$ particles. Shukla et al. (2011b and 2013) used turbulence Reynolds stress model and large-eddy simulation and injected 10800 particles. The authors simulated large number of particles, this may be the reason for the difference between simulation one-way and two-way coupling. In this study was injected 900 particles.

Figure 4 show the collection efficiency (\%) for particle of 1 to $5 \mu \mathrm{m}$. It is observed that the numerical values are close to the experimental data (Zhao et al., 2004). The numerical data, of the 
collection efficiency are in very good agreement with the experimental data. The collection efficiency obtained with one-way coupling are slightly lower compared to simulation with two-way coupling. Like tendency was to be observed by Derksen et al. (2008). The simulation of the mean tangential and axial velocity (Figures 2 and 3) were not observed differences between simulation with one-way coupling and two-way coupling.

In Figure 4 were observed differences between simulation with one-way and two-way coupling the simulation. Simulation of the collection efficiency with one-way coupling gives lower values compared to two-way coupling. Though the gas-solid flow injected in the cyclone is diluted, it is possible to observed small difference between the results obtained by the one-way coupled and the two-way coupled when is simulated the collection efficiency for particles.

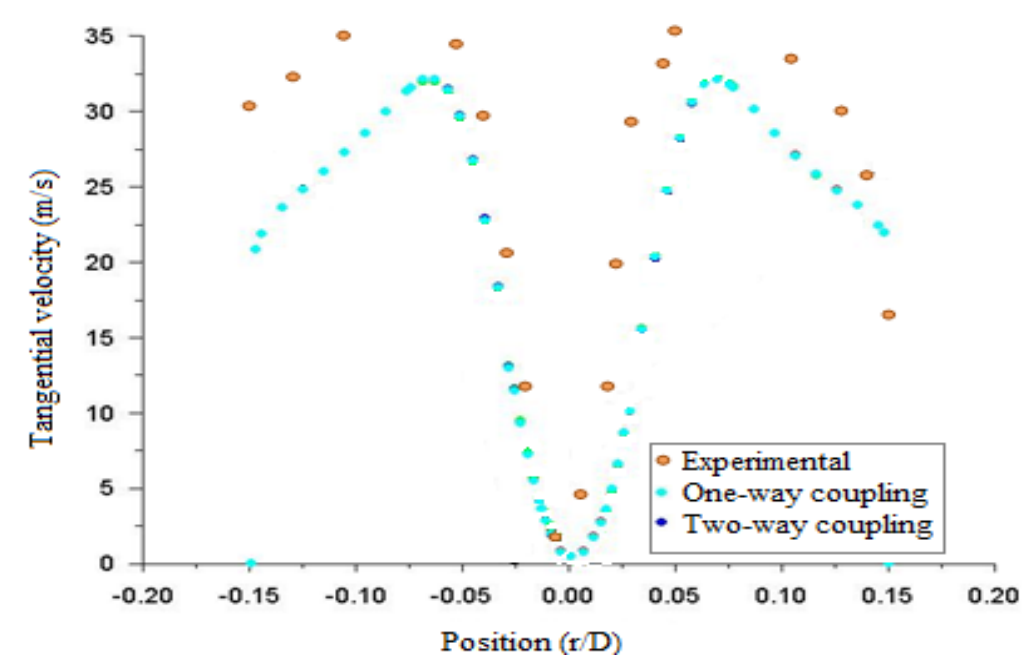

Figure 2 - The mean tangential velocity.

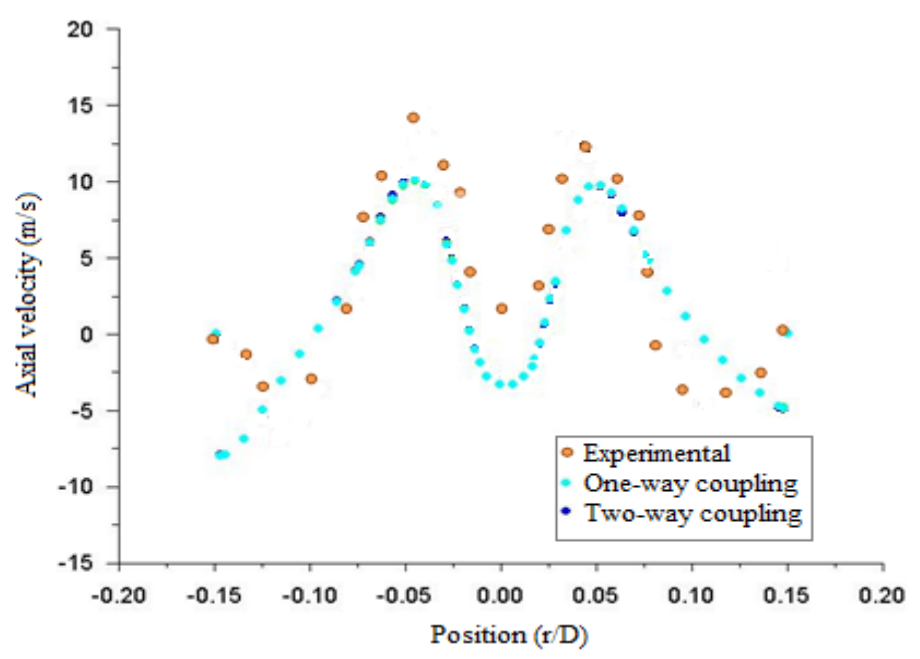

Figure 3 - The mean axial velocity. 


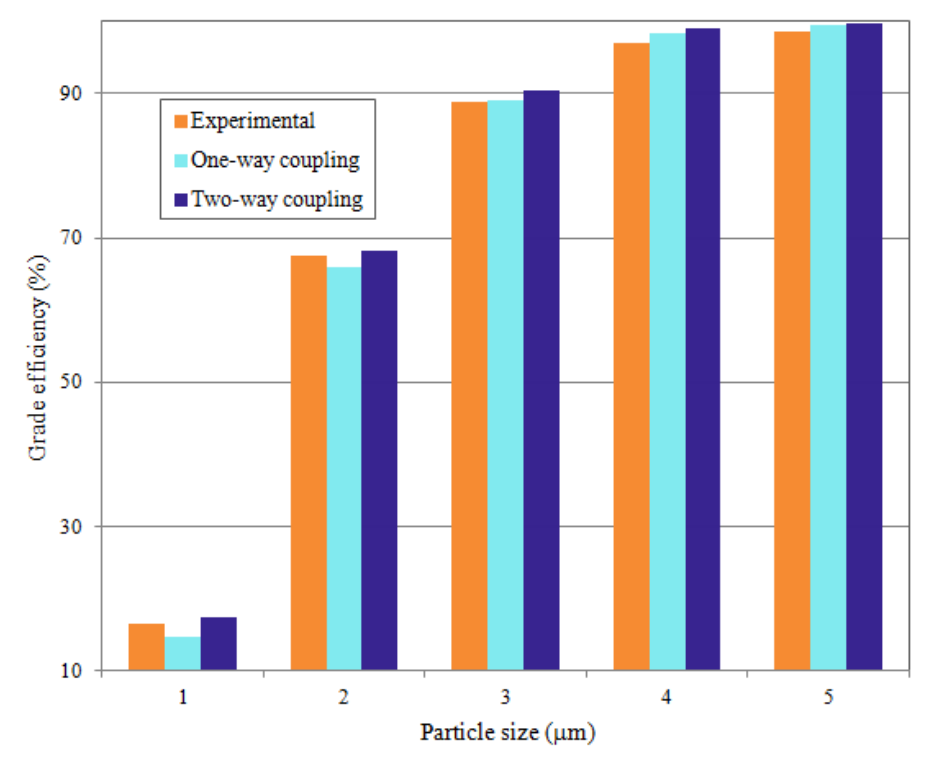

Figure 4 - Efficient of collection.

\section{CONCLUSIONS}

The simulation of gas - solid flow dilute in the cyclone was performed considering time transient and Reynolds Stress turbulent model. We studied the behavior of the particle in the cyclone considering one-way coupling and two-way coupling.

It was observed that the simulation with one-way coupling and two-way couplings exhibit the same tendency, the presence of particles did not change the profile of the tangential and axial velocity. The numerical results are close to the experimental data obtained by Zhao et al. (2004).

Though the gas-solid flow injected in the cyclone is diluted, it is possible to observed small difference between the results obtained by the one-way coupled and the two-way coupled whem is simulated the collection efficiency for particles of size 1 to $5 \mu \mathrm{m}$.

\section{ACKNOWLEDGEMENTS}

The authors wish to thank to the Brazilian agency $\mathrm{CNPq}$ (Conselho Nacional de Desenvolvimento Científico e Tecnológico) for the financial support.

\section{REFERENCES}

Crowe, C. T., Schwarzkopf, J. D., Sommerfeld, M., Tsuji, Y., 2012. Multiphase Flows with Droplets and Particles, Boca Raton, FL: CRC Press, Second Edition.

Derksen, J. J., 2003. "Separation Performance Prediction of a Stairmand high-efficiency cyclone" AIChE Journal. Vol. 49, p. 1359-1371.

Derksen, J. J., Sundaresan, S. and van den Akker, H. E. A., 2006. "Simulation of mass-loading effects in gas-solid cyclone separator" Powder Technology, Vol. 163, p. 59-68.

Derksen, J. J., van den Akker, H. E. A., Sundaresan, S., 2008. "Two-way coupled large-eddy simulation of gas-solid flow in cyclone separator" AIChE, Vol. 54, No. 4, p. 872-885.

Elghobashi, S., 1991. "On Predicting Particle-Laden Turbulent Flows: direct simulation and closure models". Applied Scientific Research, Vol.48, p. 301-314. 
Fluent User Manual.

Gimbun, J., 2008. "CFD simulation of aerocyclone hydrodynamics and performance at extreme temperature" Engineering Application of Computational Fluid Mechanical, Vol. 2, p. 22-29.

Hoekstra, A. J., Derksen, J. J., Van Den Akker, H. E. A., 1999. "An experimental and numerical study of turbulent swirling flow in gas cyclone" Chemical Engineering Science, Vol. 54, p. 20552065.

Hoffmann, A.C. and Stein, L.E., 2002. Gas Cyclones and Swirl Tubes: Principles, Design and Operation, Springer-Verlag, Heidelberg, NY, US.

Mallouppas, G., Wachem, B. V., 2013. "Large eddy simulation of turbulent particle-laden channel flow", International Journal of Multiphase Flow, Vol. 54, p. 65-75.

Loth, E., 2003. Computational Fluid Dynamics of Bubbles, Drops and Particles. Cambridge University Press.

L'vov, V. S., Ooms, G., Poyalov, A., 2003 "Effect of particle inertia on turbulence in a suspension" Physical Review, Vol. 67, p. 1-21.

Shukla, S. K., Shukla, P., Ghosh, P., 2011a. "Evaluation of numerical schemes for dispersed phase modeling of cyclone separators", Engineering Applications of Computational Fluid Mechanics, Vol 5, p. 235-246.

Shukla, S. K., Shukla, P., Ghosh, P., 2011b. "Evaluation of numerical schemes using different simulation methods for the continuous phase modeling of cyclone separator", Advanced Powder Technology, Vol 22, p. 209-219.

Shukla, S. K., Shukla, P., Ghosh, P., 2013. "The effect of modeling of velocity fluctuation on prediction of collection efficiency of cyclone separator", Applied Mathematical Modeling, Vol. 37, p. 5774-5789.

Tennekes H., Lumley, J. L., 1972. A First Course in Turbulence. Cambridge, MA: MIT Press.

Utikar, R., Darmawan, N., Tade, M., Li, Q., Evans, G., Glenny, M. and Pareek, V., 2010. Computational Fluid Dynamic. Hyoung Woo OH.

Wan, G., Sun, G., Xue, X., and Shi, M., 2008. "Solid concentration simulation of different size particles in a cyclone separator", Powder Technology, Vol. 183, p.94-104.

Zhao, B., Shen, H., Kang, Y., 2004. "Development of a symmetrical spiral inlet to improve cyclone separator performance", Powder Technology, Vol. 145, p. 47-50.

Zhao, B., Su, Y., Zhang, J., 2006. "Simulation of gas flow pattern and efficiency in cyclone with conventional single and spiral double inlet con configuration" IChem, Vol. 84, p. 1158-1165.

\section{RESPONSIBILITY NOTICE}

The authors are the only responsible for the printed material included in this paper. 